\title{
TOTAL CURVATURE AND AREA OF CURVES WITH CUSPS AND OF SURFACE MAPS
}

\author{
TOBIAS EKHOLM and FRANK KUTZSCHEBAUCH*
}

\begin{abstract}
A curvature-area inequality for planar curves with cusps is derived. Using this inequality, the total (Lipschitz-Killing) curvature of a map with stable singularities of a closed surface into the plane is shown to be bounded below by the area of the map divided by the square of the radius of the smallest ball containing the image of the map. This latter result fills the gap in Santaló's [7] proof of a similar estimate for surface maps into $\mathrm{R}^{n}, n>2$.
\end{abstract}

\section{Introduction}

Let $f: S \rightarrow \mathrm{R}^{2}$ be a smooth generic map of a closed surface to the plane. The total curvature $K(f)$ of $f$ equals $\pi$ times the average over all unit vectors $v \in \mathrm{R}^{2}$ of the number of critical points of the function $x \mapsto\langle f(x), v\rangle, x \in S$, where $\langle$,$\rangle is the standard inner product on \mathbf{R}^{2}$. The area of $f$, Area $(f)$ is its mapping area. We show that if the image of $f$ is contained in a disk of radius $\epsilon>0$ then

$$
K(f) \geq \frac{\operatorname{Area}(f)}{\epsilon^{2}},
$$

see Theorem 4.2. In order to prove (1), we establish a curvature-area estimate for plane curves with cusps in Section 3 (which we believe is of independent interest).

Combining (1) with integral geometric methods we derive similar inequalities for surface maps into $\mathrm{R}^{n}$ for arbitrary $n>1$. This method was originally used by Santaló [7] in order to establish these in the case when $f: S \rightarrow \mathbf{R}^{n}$, $n>2$, is an immersion. However, as pointed out by Chakerian [8], [9], Santaló's proof is incomplete. The point overlooked is the fact that the critical values of a plane-projection of an immersion does not generally form an immersed curve but rather a curve with cusps. (The same mistake appears in an

\footnotetext{
* TE is a research fellow of the Royal Swedish Academy of Sciences sponsored by the Knut and Alice Wallenberg foundation. FK is partially supported by the Swedish research council.

Received August 11, 2003; in revised form February 17, 2004.
} 
earlier paper of Fáry [6].) The present paper thus completes Santaló-Fáry's integral geometric proof, taking also cusps into consideration.

During the preparation of this manuscript, we were informed that the counterpart of (1) for surface immersions into $\mathrm{R}^{3}$ had been proved with a different method by Aminov [2] (see also [3], Corollary 6.22, p. 50.). This method is specific for immersions into $\mathrm{R}^{3}$. Since projections into 3-dimensional subspaces of surface immersions into $\mathrm{R}^{n}, n>3$, generically are not immersions (they have certain singularities, so called Whitney umbrellas), this result is not immediately applicable in the integral geometric arguments used in the proof of Theorem 4.4 .

We thank Alexandru Oancea for informing us about the proof of the counterpart of (1) for surface immersions into 3-space in [3].

We also thank the referee for useful suggestions and in particular, for pointing out mistakes in an earlier version of the proof of Lemma 3.5.

\section{Total curvature and generic singularities of surface maps}

In this section we first introduce the notion of total curvature of a map and then discuss generic singularities of maps between surfaces. The material presented here is well-known and we refer the reader to [4] for more information on the first subject and to [1] for the second.

\subsection{Classical total curvature and Lipschitz-Killing curvature}

Let $f: S \rightarrow \mathrm{R}^{3}$ be an immersion of a surface $S$. As mentioned in the introduction, the total curvature of $f$ is the integral of the absolute value of the Gauss curvature over the surface. It is well known that the total curvature is equal to the area of the image of the Gauss map $G: S \rightarrow \mathrm{S}^{2}$ corresponding to $f$ (here $\mathrm{S}^{2}$ is the unit sphere in $\mathrm{R}^{3}$ with its standard area form),

$$
K(f)=\operatorname{Area}(G)
$$

This last quantity can also be computed by integrating the multiplicity of the map $G$ over the unit sphere. Translating back to the original immersion we find that the total curvature of $f$ equals the average, over all directions in $\mathrm{R}^{3}$, of the number of critical points of the corresponding height functions. More precisely, if $v \in \mathrm{S}^{2}$ then the height function in direction $v$ is $h_{v}: S \rightarrow \mathrm{R}$, $s \mapsto\langle f(s), v\rangle$, where $\langle$,$\rangle denotes the standard inner product on \mathrm{R}^{3}$. If $c(v)$ denotes the number of critical points of $h_{v}$ then

$$
K(f)=\frac{1}{2} \int_{v \in \mathrm{S}^{2}} c(v) d v
$$


where $d v$ is the area element induced on the unit sphere $\mathrm{S}^{2}$ from the Euclidean metric in $R^{3}$. (Note that the area of $S^{2}$ is $4 \pi$.)

The expression (3) for the total curvature applies not only to immersions but to more general maps. Therefore we take this as our definition. Moreover, (3) generalizes immediately to maps $f: S \rightarrow \mathrm{R}^{n}$ for $n \neq 3$. In this case the total curvature (or Lipschitz-Killing curvature) of a map $f: S \rightarrow \mathrm{R}^{n}$ is

$$
K(f)=\frac{1}{2} \int_{v \in S^{n-1}} c(v) d v,
$$

where $d v$ is the standard volume form on the unit sphere $\mathrm{S}^{n-1} \subset \mathrm{R}^{n}$. If $n>3$ and if $f: S \rightarrow \mathrm{R}^{n}$ is an immersion then also (2) has a counterpart. In this case the Gauss-map is naturally defined on the geometric unit normal bundle of $f$ (i.e., the bundle with fiber over $s \in S$ consisting of unit vectors in $\mathbf{R}^{n}$ orthogonal to the tangent plane $d f\left(T_{s} S\right)$ ) and again $K(f)$ equals the volume of the Gauss map.

It will also be convenient to normalize $K(f)$ differently. We write $\hat{K}(f)$ for $K(f)$ divided by the volume of the $(n-1)$-sphere. (That is, in (4), the rotational invariant measure with total mass 1 is used instead of $d v$.)

\subsection{Stable singularities of maps $f: S \rightarrow \mathbf{R}^{2}$}

Let $U \subset \mathrm{R}^{m}$ be open and let $g: U \rightarrow \mathrm{R}^{n}$ be a $C^{k}$-map, $k>1$. We say that $g$ is stable if there exists an $\epsilon>0$ such that any $C^{k}$-map $h: U \rightarrow \mathrm{R}^{n}$ of $C^{k}$-distance less than $\epsilon$ from $g$ is conjugate to $g$ via left-right action of $C^{k}$ diffeomorphisms. That is, $h=\phi \circ g \circ \psi$ for diffeomorphisms $\phi: U \rightarrow U$ and $\psi: \mathrm{R}^{n} \rightarrow \mathrm{R}^{n}$. We say that a $C^{k}$-map $f: M \rightarrow \mathrm{R}^{n}$ of a smooth manifold $M$ has stable singularities if $f$ is locally stable.

Any $C^{3}$-map $S \rightarrow \mathrm{R}^{2}$ of a surface $S$ can be perturbed with an arbitrarily small perturbation to a map with stable singularities. Let $f: S \rightarrow \mathrm{R}^{2}$ be a $C^{3}$ map with stable singularities. Then there exists local $\left(C^{3}\right.$-)coordinates $(u, v)$ around any point $s \in S$ and $(x, y)$ around $f(s) \in \mathbf{R}^{2}$ so that the map has one of the following local forms.

(a) A regular point, $f(u, v)=(u, v)$.

(b) A fold point, $f(u, v)=\left(u^{2}, v\right)$.

(c) A cusp point, $f(u, v)=\left(u^{3}+u v, v\right)$.

In particular, the preimage of the singular values of $f$ is a $\left(C^{2}\right.$-smooth) 1-dimensional submanifold $\tilde{\Sigma}$ of $S$. Along $\tilde{\Sigma}$ the line field $\operatorname{Ker}(d f)$ is defined and it is tangent to $\tilde{\Sigma}$ exactly at the cusp points. The curve $\Sigma=f(\tilde{\Sigma})$ is immersed away from the cusps. We refer to it as the fold curve of $f$. 
If $f: S \rightarrow \mathbf{R}^{2}$ is a map with stable singularities then all critical points of any height function $h_{v}, v \in \mathrm{S}^{1}$ lies in $\tilde{\Sigma}$. It follows that the total curvature of $f$ equals the total curvature of its fold curve $\Sigma$. More precisely, if $\Sigma_{1} \cup \ldots \cup \Sigma_{m}$ is a subdivision of $\Sigma$ into immersed segments then

$$
K(f)=\sum_{j=1}^{m} \int_{\Sigma_{j}}|\kappa| d s,
$$

where $\kappa$ is the curvature function along $\Sigma_{j}$ and $d s$ is the arc length element.

\section{Curvature and area of curves with cusps}

In this section we derive the basic area-curvature estimate for a curve with cusps and with image in a disk. We first define basic concepts and the class of curves in which the curvature-area estimate will be proved. (Note that the fold curve of a map of a surface to a plane with stable singularities can be approximated arbitrarily well by curves in this class.) Then, in a sequence of lemmas, we show how to simplify the curves without decreasing the relevant curvature-area difference. In the final subsection we prove the estimate for these simple curves.

\subsection{Basic definitions}

Let $\Gamma$ be a piecewise regular closed curve in the plane. That is, $\Gamma=\cup_{j=1}^{m} \Gamma_{m}$ is a cyclically ordered union of finitely many immersed curves $\Gamma_{j}$ with arc length parameterizations $c_{j}:\left[0, L_{j}\right] \rightarrow \mathrm{R}^{2}, c_{j}\left(L_{j}\right)=c_{j+1}(0)$, and $c_{m}\left(L_{m}\right)=c_{1}(0)$.

Recall that the tangent cone of a subset $E \subset \mathrm{R}^{n}$ at $p \in E$ is defined as

$$
\{r \in \mathrm{R}: r \geq 0\} \cdot\left\{\bigcap_{\epsilon>0} \text { Closure }\left\{\frac{x-p}{|x-p|}: x \in E, 0<|x-p|<\epsilon\right\}\right\}
$$

If $t \in\left(0, L_{j}\right)$ is an interior point of one of the parameterizing intervals of $\Gamma$ then we define the tangent cone $T_{c_{j}(t)} \Gamma$ of $\Gamma$ at $c_{j}(t)$ as the tangent cone of $c_{j}(U)$ at $c_{j}(t)$, where $U \subset\left(0, L_{j}\right)$ is any open interval around $t$ on which $c_{j}$ is an embedding. If $t=L_{j}$ is an end point of $\left[0, L_{j}\right]$ we define $T_{c_{j}\left(L_{j}\right)} \Gamma$ as the tangent cone of $c_{j}(U) \cup c_{j+1}(V)$ where $U=\left(L_{j}-\delta, L_{j}\right]$ and $V=[0, \delta)$ and $\delta>0$ is small enough for both $c_{j} \mid U$ and $c_{j+1} \mid V$ to be embeddings.

A point $p=c_{j}\left(L_{j}\right)=c_{j+1}(0)$ on $\Gamma$ is a vertex of $\Gamma$ if the tangent cone of $\Gamma$ at $p$ is not a straight line.

A choice of orientation on one of the $\Gamma_{j}$ induces an orientation on $\Gamma$ and hence distinguishes positive unit tangent vector fields $T_{j}$ along $\Gamma_{j}$. Instead of working with orientations we will often use coorientations: a coorientation of $\Gamma$ is a collection of normal vector fields $N_{j}$ defined along each $\Gamma_{j}$ which 
are orthogonal to the corresponding unit tangent vector fields $T_{j}$ coming form some orientation of $\Gamma$, and such that the orientations on $\mathrm{R}^{2}$ induced by $\left(N_{j}, T_{j}\right)$ agrees for all $j$. Thus a coorientation induces an orientation of $\Gamma$.

Let $(x, y)$ be orthogonal coordinates on $\mathrm{R}^{2}$ and let $\alpha$ be the 1 -form $\alpha=$ $\frac{1}{2}(x d y-y d x)$. Then $d \alpha=d x \wedge d y$ is the area from on $\mathrm{R}^{2}$.

Definition 3.1. The area-content $A(\Gamma)$ of a cooriented piecewise regular curve $\Gamma$ is

$$
A(\Gamma)=\frac{1}{2} \int_{\Gamma}(x d y-y d x),
$$

where $\Gamma$ is endowed with the orientation induced by the coorientation.

Let $\Gamma$ be a piecewise regular curve and let $p$ be a vertex of $\Gamma$. The vertex $p$ will be called non-degenerate if the tangent cone $T_{p} \Gamma$ of $\Gamma$ at $p$ consists of two distinct half-lines at $p$. Note that a piecewise regular curve with non-degenerate vertices is a local (continuous) embedding.

Let $p=c_{j}\left(L_{j}\right)=c_{j+1}(0)$ be a non-degenerate vertex of $\Gamma$ and let $v_{1}$ and $v_{2}$ be unit vectors at $p$ in the directions of the half-lines in $T_{p} \Gamma$. We say that a coorientation of $\Gamma$ is convex (concave) at $p$ if $\left\langle N_{j}\left(L_{j}\right)+N_{j+1}(0), v_{1}+v_{2}\right\rangle<0$ $\left(\left\langle N_{j}\left(L_{j}\right)+N_{j+1}(0), v_{1}+v_{2}\right\rangle>0\right)$, where $\langle$,$\rangle is the standard inner product$ on $\mathrm{R}^{2}$.

Let $\Gamma$ be a closed piecewise regular curve with non-degenerate vertices and let $p=c_{j}(t) \in \Gamma$. We say that $\Gamma$ is locally convex (locally concave) if all its corners are convex (concave) and the curvature function of each regular point of $\Gamma$ is non-negative (non-positive).

A fold-approximation is a cooriented piecewise regular curve $\Gamma$ with nondegenerate vertices, with each vertex labeled as either a wedge or a corner, and such that the coorientation of $\Gamma$ is convex at each wedge.

If $\Gamma$ is a fold-approximation we write $\operatorname{Wdg}(\Gamma)$ and $\operatorname{Crn}(\Gamma)$ for its sets of wedges and of corners, respectively. If $p$ is a vertex of $\Gamma$ then let $i(p)$ denote the angle between the half-lines in the tangent cone $T_{p} \Gamma$ and let $e(p)=\pi-i(p)$. (So that, $i(p)$ and $e(p)$ are the interior- respectively exterior angles at $p$.)

Definition 3.2. The total curvature $K(\Gamma)$ of a fold-approximation $\Gamma=$ $\cup_{j} \Gamma_{j}$ is

$$
K(\Gamma)=\sum_{j} \int_{\Gamma_{j}}|\kappa| d s+\sum_{p \in \operatorname{Crn}(\Gamma)} e(p)+\sum_{p \in \mathrm{Wdg}(\Gamma)} i(p),
$$

where $\kappa$ denotes the curvature function of $\Gamma_{j}$. 


\subsection{The estimate}

In this section we present a sequence of lemmas which taken together prove the following result.

THEOREM 3.3. If $\Gamma$ is a fold-approximation lying inside a disk of radius $\epsilon>0$ then

$$
K(\Gamma)-\frac{2 A(\Gamma)}{\epsilon^{2}} \geq 0
$$

To prove theorem 3.3 we make two observations. First, a piecewise regular curve $\Gamma=\cup_{j} \Gamma_{j}$ will be called piecewise linear or simply PL if each $\Gamma_{j}$ is a line segment. The well-known approximation result relating the total curvature of a curve to the total curvature of polygons inscribed in it, see [5], shows that it is enough to prove (6) for PL fold-approximations. Second, note that scaling by $\epsilon$ does not change the total curvature of a fold-approximation, whereas it multiplies the area-content by $\epsilon^{2}$. Therefore, it is sufficient to prove Theorem 3.3 for $\epsilon=1$.

Thus, we will assume that $\Gamma$ is a PL fold-approximation contained in the unit disk $D$ and we prove the estimate $K(\Gamma)-2 A(\Gamma) \geq 0$.

In order to prove this estimate we will use several lemmas presented below. The purpose of these lemmas are to deform a given PL fold-approximation into a fold-approximation with certain special properties without increasing $K-2 A$. The proof of Theorem 3.3 will then be completed by proving it for fold-approximations which have these special properties.

Lemma 3.4. Let $\Gamma$ be a PL fold-approximation in D. Then there exists a PL fold-approximation $\Gamma_{0}$ in $D$ with all its wedges on the boundary $\partial D$ and such that

$$
K\left(\Gamma_{0}\right)-2 A\left(\Gamma_{0}\right) \leq K(\Gamma)-2 A(\Gamma) .
$$

Proof. Let $p$ be a wedge on $\Gamma$ which does not lie on $\partial D$. Let $L$ be the halfline at $p$ generated by the unit vector $v$ which forms the same angle with the two half-lines in the tangent cone $T_{p} \Gamma$ and such that for any vector $w \in T_{p} \Gamma$, $\langle v, w\rangle \leq 0$. Then $L$ meets $\partial D$ in some point $q$. Let $L_{1}$ and $L_{2}$ be half-lines at $q$ pointing inside $D$ which form angles $\frac{\alpha}{2}$ and $-\frac{\alpha}{2}$ with $L$. Then for $\alpha>0$ small enough $L_{1}$ and $L_{2}$ meet $\Gamma$ at two points $p_{1}$ and $p_{2}$ which are close to $p$. Construct the curve $\Gamma^{\prime}$ by replacing the polygonal arc $p_{1} p p_{2}$ in $\Gamma$ by the polygonal arc $p_{1} q p_{2}$, let $q$ be a wedge and $p_{1}, p_{2}$ be corners.

It is then easy to check that $K\left(\Gamma^{\prime}\right)=K(\Gamma)$, see Figure 1. Since the coorientation of $\Gamma$ at $p$ is convex there is a unique coorientation $\Gamma^{\prime}$ in which $q$ is convex and $A\left(\Gamma^{\prime}\right) \geq A(\Gamma)$. 


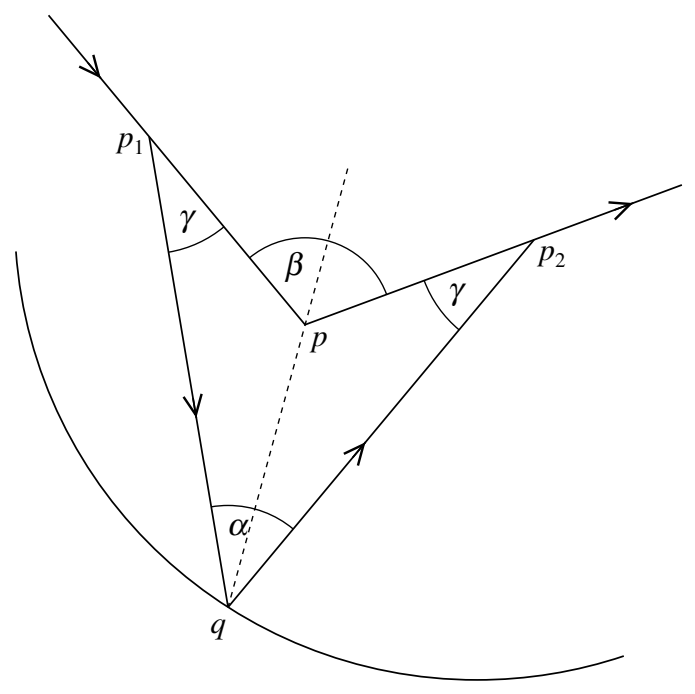

FiguRE 1. $\Delta K=-\beta+\alpha+2 \gamma=0, \Delta A>0$.

Let $\Gamma_{0}$ be the PL fold-approximation which is obtained by applying the above process at each wedge not on $\partial D$.

Lemma 3.5. Let $\Gamma_{0}$ be as in Lemma 3.4. Then there exists a locally convex PL fold-approximation $\Gamma_{1}$ with each wedge on the boundary, such that the coorientation is convex at each vertex of $\Gamma_{1}$, and such that

$$
K\left(\Gamma_{1}\right)-2 A\left(\Gamma_{1}\right) \leq K\left(\Gamma_{0}\right)-2 A\left(\Gamma_{0}\right) .
$$

Proof. We make $\Gamma_{0}$ locally convex using an inductive procedure. Assume first that there exists three consecutive vertices $p, q$, and $r$ on $\Gamma_{0}$ such that $p$ is a convex wedge or corner and $q$ is a concave corner. Let $\Gamma^{\prime}$ be the PL curve obtained from $\Gamma_{0}$ by replacing the segments $p q$ and $q r$ by the segment $p r$. Then $\Delta K=K\left(\Gamma^{\prime}\right)-K\left(\Gamma_{0}\right) \leq 0$ and $\Delta A=A\left(\Gamma^{\prime}\right)-A\left(\Gamma_{0}\right)>0$. To see this confer Figure 2.

Let $s$ be the vertex immediately after $r$ on $\Gamma_{0}$. Since $\Gamma_{0}$ is convex at $p, s$ and $p$ are distinct vertices of $\Gamma_{0}$. The curve $\Gamma^{\prime}$ is a fold approximation except when the vertex $s$ lies on the half-line $l$ starting at $r$ and passing through $p$. If $s \notin l$ then take $\hat{\Gamma}=\Gamma^{\prime}$. If $s \in l$ then let $\hat{\Gamma}$ be the curve obtained from $\Gamma^{\prime}$ by moving $s$ slightly off $l$ in such a way that its corner at $r$ is convex and so that $K(\hat{\Gamma})-2 A(\hat{\Gamma})<K\left(\Gamma_{0}\right)-2 A\left(\Gamma_{0}\right)$. (Even though moving $s$ might increase $K-2 A$, the latter condition is met provided the deformation is sufficiently small since $K\left(\Gamma^{\prime}\right)-2 A\left(\Gamma^{\prime}\right)<K\left(\Gamma_{0}\right)-2 A\left(\Gamma_{0}\right)$.) Note that the number of vertices of $\hat{\Gamma}$ is strictly smaller than the number of vertices of $\Gamma_{0}$. 


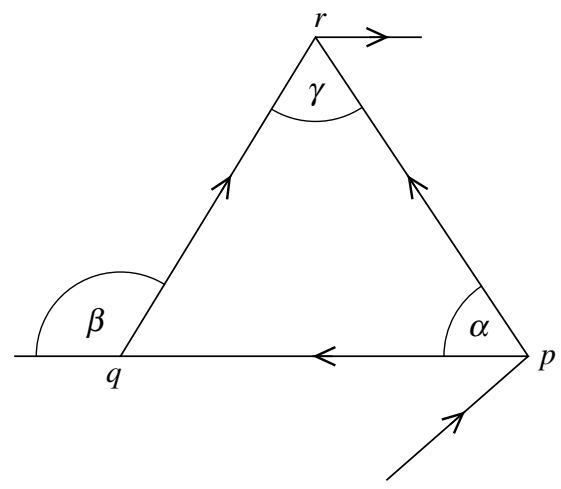

FIGURE 2. $\Delta K \leq \alpha+\gamma-\beta=0, \Delta A>0$.

Assume secondly that $\Gamma_{0}$ has only concave vertices. (In particular, all of its vertices must be corners since, by definition, the wedges of a fold approximation are convex.) If $\Gamma_{0}$ is an embedded curve then its local concavity implies it is negatively oriented and hence $A\left(\Gamma_{0}\right)<0$. In this case we may take $\hat{\Gamma}$ as the empty curve and $K(\hat{\Gamma})-2 A(\hat{\Gamma})=0<K\left(\Gamma_{0}\right)-2 A\left(\Gamma_{0}\right)$.

In order to deal with the case when $\Gamma_{0}$ is not an embedding we introduce the following notation. A PL curve $f: S^{1} \rightarrow \mathrm{R}^{2}$ is generic if all its vertices are nondegenerate and all its multiple points are transverse intersections between two edges. An arbitrary PL curve with non-degenerate vertices can be deformed into a generic PL curve by an arbitrary small deformation which does not increase the number of vertices. A double point $s$ of a generic PL curve $f: S^{1} \rightarrow$ $\mathrm{R}^{2}$ is innermost if $f^{-1}(s)$ bounds an arc $A \subset S^{1}$ such that the restriction of $f$ to the interior of $A$ is injective. Clearly, any generic PL curve has an innermost double point.

With this notation introduced, consider the case when $\Gamma_{0}$ is a locally concave non-injective curve. Let $p, q, r$ be consecutive vertices of $\Gamma_{0}$. Let $\Gamma^{\prime}$ be the curve obtained from $\Gamma_{0}$ by moving $q$ a small distance along the segment $q p$, starting at $q$ ending at $p$, to a new position $q^{\prime}$, see Figure 3 .

Then $\Gamma^{\prime}$ is still locally concave so $K(\Gamma)=K\left(\Gamma^{\prime}\right)$ and $A\left(\Gamma^{\prime}\right)=A(\Gamma)+$ $\operatorname{Area}\left(T\left(r q q^{\prime}\right)\right)>A(\Gamma)$, where $T\left(r q q^{\prime}\right)$ is the triangle with corners $r, q$, and $q^{\prime}$. Let $\Gamma^{\prime \prime}$ be a small perturbation of $\Gamma^{\prime}$ such that $\Gamma^{\prime \prime}$ is generic and locally concave, so that $\Gamma^{\prime \prime}$ has the same number of vertices as $\Gamma^{\prime}$, and so that $K\left(\Gamma^{\prime \prime}\right)-$ $2 A\left(\Gamma^{\prime \prime}\right)<K\left(\Gamma_{0}\right)-2 A\left(\Gamma_{0}\right)$. (Such a curve exists since locally concavity is an open condition.) Let $s$ be an innermost self intersection point of $\Gamma^{\prime \prime}$. Then $\Gamma^{\prime \prime}$ can be written as a union $\Gamma^{\prime \prime}=\hat{\Gamma}_{1} \cup \hat{\Gamma}_{2}$, where $\hat{\Gamma}_{j}, j=1,2$, is a closed curve with a vertex at $s$. Since $s$ was an innermost double point of $\Gamma^{\prime \prime}$ at least one of the curves $\hat{\Gamma}_{j}, j=1,2$ is embedded. Choose notation so that $\hat{\Gamma}_{2}$ is 


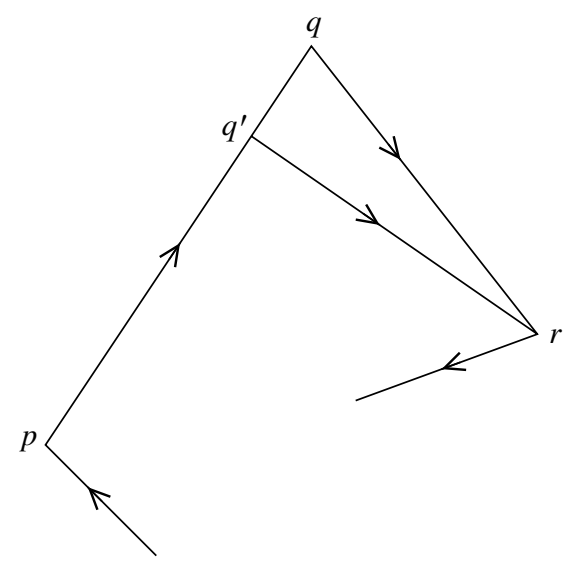

Figure 3. Deforming $\Gamma_{0}$ to $\Gamma^{\prime}$.

an embedding. Since $\hat{\Gamma}_{2}$ is a closed curve, $K\left(\hat{\Gamma}_{2}\right) \geq 2 \pi$, and since $\hat{\Gamma}_{2}$ is a negatively oriented embedded curve, $A\left(\hat{\Gamma}_{2}\right)<0$. Let $\delta, 0<\delta<\pi$ be the exterior angle of $\hat{\Gamma}_{2}$ at $s$. Then the exterior angle of $\hat{\Gamma}_{1}$ at $s$ equals $\delta$ as well. We have

$$
\begin{aligned}
& K\left(\hat{\Gamma}_{1}\right)=K\left(\Gamma^{\prime \prime}\right)-\left(K\left(\hat{\Gamma}_{2}\right)-\delta\right)+\delta \leq K\left(\Gamma^{\prime \prime}\right)-2(\pi-\delta)<K\left(\Gamma^{\prime \prime}\right), \\
& A\left(\hat{\Gamma}_{1}\right)=A\left(\Gamma^{\prime \prime}\right)-A\left(\hat{\Gamma}_{2}\right)>A\left(\Gamma^{\prime \prime}\right) .
\end{aligned}
$$

Thus, taking $\hat{\Gamma}=\hat{\Gamma}_{1}$ we find $K(\hat{\Gamma})-2 A(\hat{\Gamma})<K\left(\Gamma_{0}\right)-2 A\left(\Gamma_{0}\right)$. Moreover, since $\hat{\Gamma}_{2}$ has at least three vertices we find that the number of vertices of $\hat{\Gamma}$ is smaller than the number of vertices of $\Gamma_{0}$.

In conclusion: the above argument constructs, from any PL fold approximation $\Gamma_{0}$ as in the statement of the lemma with at least one concave corner, another PL fold approximation $\hat{\Gamma}$ which has the properties of $\Gamma_{0}$ in the statement of the lemma, which satisfies $K(\hat{\Gamma})-2 A(\hat{\Gamma})<K\left(\Gamma_{0}\right)-2 A\left(\Gamma_{0}\right)$, and which has at least one vertex less than $\Gamma_{0}$. Applying this procedure inductively until there are no concave corners, we arrive at a locally convex curve $\Gamma_{1}$ with properties as desired.

Lemma 3.6. Let $\Gamma_{1}$ be as in Lemma 3.5. Then there exists a locally convex fold-approximation with the following properties. If $\Gamma_{1}$ has wedges then the wedges of $\Gamma_{2}$ are at the same points and have the same angles as those of $\Gamma_{1}$, and if $C$ is a part of $\Gamma_{2}$ connecting two wedges $p_{1}$ and $p_{2}$ then $C$ has one of the following forms

(a) $C$ is a line segment. 
(b) C consists of two line segments and has one corner where the coorientation is convex.

(c) $C=L_{1} \cup S \cup L_{2}$ where $L_{1}$ is a line segment from $p_{1}$ to a point $q_{1} \in \partial D$, $S$ is a positively oriented curve in the boundary $\partial D$ connecting $q_{1}$ to a point $q_{2}$ in the boundary, and $L_{2}$ is a line segment connecting $q_{2}$ to $p_{2}$. The coorientations at the corners $q_{1}$ and $q_{2}$ are convex.

If $\Gamma_{1}$ has no wedges then then $\Gamma_{2}$ is a curve in the boundary $\partial D$. Moreover,

$$
K\left(\Gamma_{2}\right)-2 A\left(\Gamma_{2}\right) \leq K\left(\Gamma_{1}\right)-2 A\left(\Gamma_{1}\right) .
$$

Proof. We will use the following notation in the proof below: if $\gamma$ is a piecewise regular curve then $\gamma=\cup_{j} \gamma_{j}$ where $\gamma_{j}$ are regular curves connecting the vertices of $\gamma$. We call $\gamma_{j}$ the regular segments of $\gamma$.

Consider first the case when $\Gamma_{1}$ has wedges. We deform $\Gamma_{1}$ to a curve of the desired form using an inductive procedure. In order to do so, let $\Gamma$ be a locally convex fold approximation in $D$ with all its wedges on the boundary and such that each regular segment of $\Gamma$ is either a curve in $\partial D$ or a straight line segment. (Note that $\Gamma_{1}$ in the formulation of the lemma fulfills these conditions. Note also that if $\Gamma$ fulfills these condition and no regular segment between two corners of $\Gamma$ is a straight line segment then $\Gamma$ has the properties of $\Gamma_{2}$ in the statement of the lemma.)

Assume that $\Gamma$ has a regular segment connecting two of its corners $q_{1}$ and $q_{2}$ which is a straight line segment. Let $l_{1}$ be the oriented tangent line to the regular segment of $\Gamma$ ending at $q_{1}$ and let $l_{2}$ be the oriented tangent line of the regular segment beginning at $q_{2}$. Let $L_{1} \subset l_{1}$ be the half-line starting at $q_{1}$ and let $L_{2} \subset l_{2}$ be the half-line ending at $q_{2}$. If $L_{1}$ and $L_{2}$ intersect at a point $q$ inside the disk then let $\hat{\Gamma}$ be the curve obtained from $\Gamma$ by replacing the line segment $q_{1} q_{2}$ by the line segments $q_{1} q$ and $q q_{2}$, see Figure 4 . If the two half-lines $L_{1}$ and $L_{2}$ do not intersect in $D$ then let $\hat{\Gamma}$ be the curve obtained from $\Gamma_{1}$ by replacing the line segment $q_{1} q_{2}$ by the curve $C$ defined as follows.

- If $q_{1} \notin \partial D$ and $q_{2} \notin \partial D$ then $C$ consists of $L_{1} \cap D$, the positively oriented arc in $\partial D$ connecting $L_{1} \cap \partial D$ to $L_{2} \cap \partial D$, and $L_{2} \cap D$, see Figure 4.

- If $q_{1} \in \partial D$ and $q_{2} \notin \partial D$ then $C$ consists of the positively oriented arc in $\partial D$ connecting $L_{1} \cap \partial D$ to $L_{2} \cap \partial D$ and $L_{2} \cap D$.

- If $q_{1} \notin \partial D$ and $q_{2} \in \partial D$ then $C$ consists of the $L_{1} \cap D$ and the positively oriented arc in $\partial D$ connecting $L_{1} \cap \partial D$ to $L_{2} \cap \partial D$.

- If $q_{1} \in \partial D$ and $q_{2} \in \partial D$ then $C$ the positively oriented arc in $\partial D$ connecting $L_{1} \cap \partial D$ to $L_{2} \cap \partial D$. 

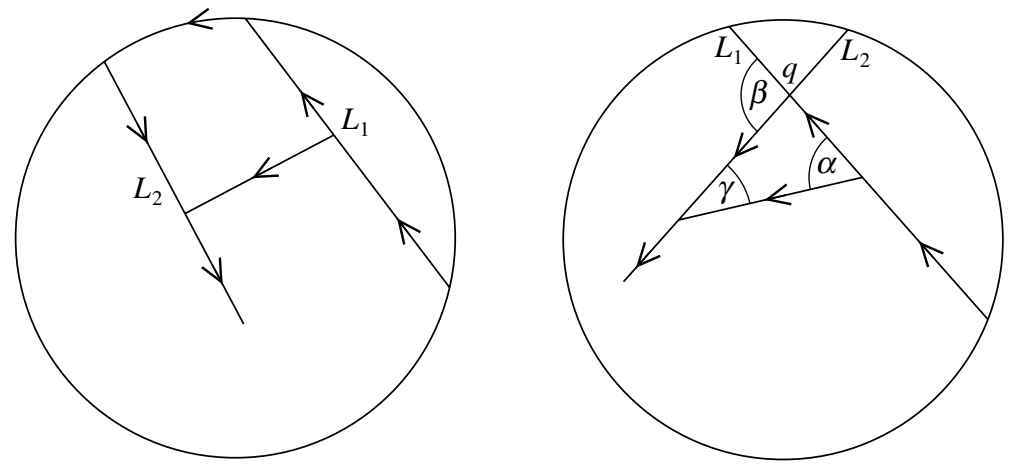

Figure 4. $\Delta K=0, \Delta A \geq 0$.

Then $\hat{\Gamma}$ is locally convex, $K(\hat{\Gamma})=K(\Gamma), A(\hat{\Gamma})>A(\Gamma)$, and the number of regular segments of $\hat{\Gamma}$ which connects two of its corners and which are straight line segments is strictly smaller than the corresponding number for $\Gamma$.

Thus, by repeating this construction we eventually (after a finite number of iterations) find a locally convex curve $\Gamma^{\prime}$ with the same wedges as $\Gamma$, with each regular segment connecting two corners being a curve in $\partial D$, and such that $K\left(\Gamma^{\prime}\right)=K(\Gamma)$ and $A\left(\Gamma^{\prime}\right) \geq A(\Gamma)$. Taking $\Gamma_{1}=\Gamma$ and $\Gamma_{2}=\Gamma^{\prime}$ finishes the proof.

\subsection{Proof of Theorem 3.3}

After the lemmas above we may assume that the fold-approximation $\Gamma$ has the form of $\Gamma_{2}$ in Lemma 3.6. From $\Gamma$ we first construct a collection of non-closed fold-approximations by removing pieces of all segments of $\Gamma$ which are of type (b) in Lemma 3.6 as follows.

Consider a segment of $\Gamma$ which has type (b) and which connects two wedges $p_{1}$ and $p_{2}$ and has a convex corner $c$ in between them. Denote the intersection of the half-line from $p_{1}$ through $c$ with the circle $\partial D$ by $q_{1}$ and the intersection of the half-line from $p_{2}$ through $c$ with the circle $\partial D$ by $q_{2}$. (If $c$ lies on the boundary then $c=q_{1}=q_{2}$.) Let $a_{1}$ denote the mid-point of the line segment $p_{1} q_{1}$ and $a_{2}$ denote the mid-point of the line segment $p_{2} q_{2}$. We replace the segment of $\Gamma$ of type (b) by the union of the line segments $p_{1} a_{1}$ and $a_{2} p_{2}$, see Figure 5 and obtain in this way a collection of non-closed curves. (For the remainder of this proof, we denote oriented line segments so that they are oriented from start-point to end-point.)

Note that even in the case $a_{1}=a_{2}=c$ this point will not be considered as a vertex (i.e., there is no curvature concentrated at that point). We just consider the non-closed curves as ending and starting at this point. Let $\Gamma^{\prime}$ denote the 


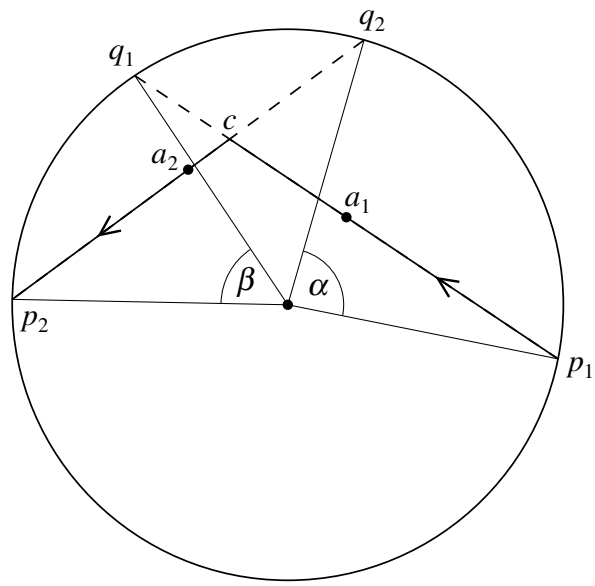

Figure 5

union of the non-closed fold-approximations obtained from $\Gamma$ by altering all segments of type (b) as described above.

We claim that

$$
K\left(\Gamma^{\prime}\right)-2 A\left(\Gamma^{\prime}\right) \leq K(\Gamma)-2 A(\Gamma)
$$

(Note that the area content $A(\gamma)$ of a non-closed curve $\gamma$, see Definition 3.1, depends on the origin of the coordinate system. In this proof we take the origin at the center of the disk $D$.) To see this, we let $\alpha$ denote the angle from the point $p_{1}$ on the circle $\partial D$ to the point $q_{2}$ on the circle $\partial D$, and we let $\beta$ denote the angle from $q_{1}$ to $p_{2}$. Then the change of total curvature is $\Delta K=-\frac{\alpha+\beta}{2}$, see Figure 5.

On the other hand, with $T(a b c)$ denoting the triangle with corners $a, b$ and $c$, the change of area is

$$
\begin{aligned}
\Delta A & =\frac{1}{2} A\left(p_{1} q_{1}\right)+\frac{1}{2} A\left(q_{2} p_{2}\right)-A\left(p_{1} c\right)-A\left(c p_{2}\right) \\
& =\frac{1}{2}\left(-A\left(p_{1} c\right)+A\left(c q_{1}\right)-A\left(c p_{2}\right)+A\left(q_{2} c\right)\right) \\
& =\frac{1}{2}\left(\operatorname{Area}\left(T\left(p_{1} q_{2} c\right)\right)-A\left(p_{1} q_{2}\right)+\operatorname{Area}\left(T\left(q_{1} p_{2} c\right)\right)-A\left(q_{1} p_{2}\right)\right) \\
& =\frac{1}{2}\left(\operatorname{Area}\left(T\left(p_{1} q_{2} c\right)\right)-\frac{\sin \alpha}{2}+\operatorname{Area}\left(T\left(q_{1} p_{2} c\right)\right)-\frac{\sin \beta}{2}\right) .
\end{aligned}
$$


We obtain

$$
\begin{aligned}
\Delta K-2 \Delta A=\frac{1}{2}( & \sin (\alpha)-\alpha)+\frac{1}{2}(\sin \beta-\beta) \\
& - \text { Area }\left(T\left(p_{1} q_{2} c\right)\right)-\text { Area }\left(T\left(q_{1} p_{2} c\right)\right) \leq 0,
\end{aligned}
$$

and the claim follows.

To finish the proof we show $K\left(\Gamma^{\prime}\right)-2 A\left(\Gamma^{\prime}\right) \geq 0$. To this end subdivide each component of $\Gamma^{\prime}$ into pieces as follows. For each straight line segment $L$ of $\Gamma^{\prime}$ between points on $\partial D$ which occurs in a segment of either type (a) or (c) in Lemma 3.6, mark its mid-point. For each curve $C$ in $\partial D$ which occurs in a segment of type (c) in Lemma 3.6, mark its mid-point. These mid-points subdivide $\Gamma^{\prime}$ into two types of curves.

(A) The curve starts at a mid-point of a straight line segment between two points on $\partial D$, follows it to a wedge on $\partial D$, leaves that wedge along a straight line segment between two points on $\partial D$, and ends at the midpoint of that segment, see Figure 6.

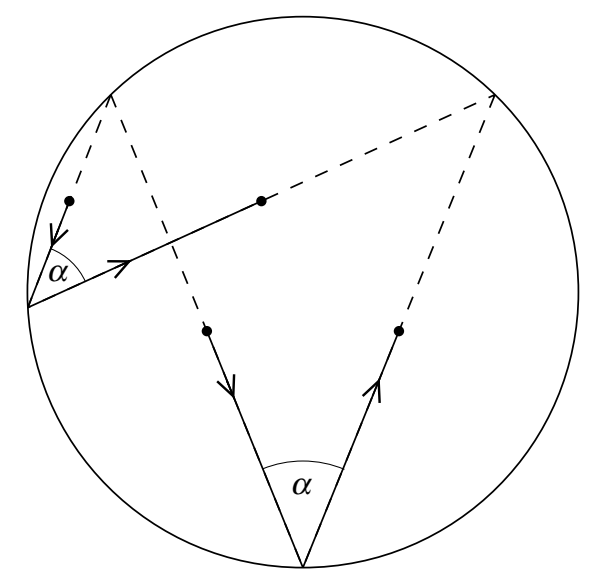

FIGURE 6

(B) The curve starts at the mid-point of a straight line segment between two points on $\partial D$, follows it to a corner on $\partial D$, leaves that corner along a positively oriented curve in the boundary $\partial D$, and ends at its mid-point. Or the other way around, i.e., starts at the mid-point of a positively oriented curve in $\partial D$, follows it to a corner on $\partial D$, leaves that corner along a straight line segment between two points on $\partial D$, and ends at its mid-point, see Figure 7. 


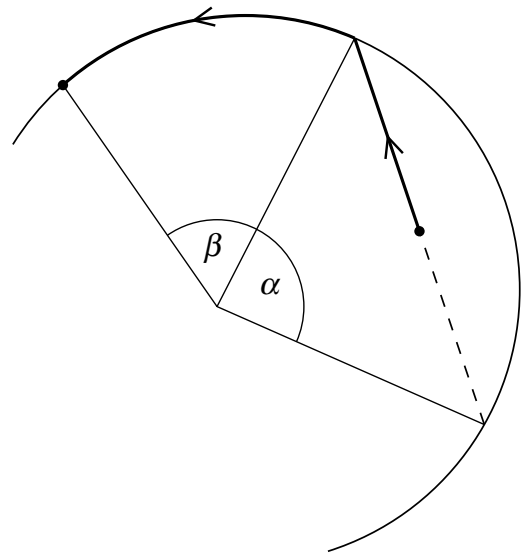

FiguRE 7

We next show that if $C$ is a curve of type (A) or (B) then $K(C)-2 A(C) \geq 0$. First, assume $C$ is of type (A) and note that the area content of half a line segment is half the area content of the whole line segment. Let $C^{\prime}$ be the curve which consists of the complete line segments (with both end points on $\partial D$ ) corresponding to the half line segments of $C$. Then $K\left(C^{\prime}\right)-A\left(C^{\prime}\right)$ does not increase as the wedge of $C^{\prime}$ is moved along $\partial D$ to the mid-point of the arc between the free end points of the line segments of $C^{\prime}$. Noting that the angle at the wedge remains constant under such a motion and that the area content is maximal in the final position, we conclude (see Figure 6) $K\left(C^{\prime}\right)-A\left(C^{\prime}\right) \geq \alpha-\sin \alpha \geq 0$ and therefore $K(C)-2 A(C) \geq 0$.

Second, assume $C$ is of type (B). If $\alpha$ denotes the length of the positively oriented arc in $\partial D$ connecting the endpoints of the straight line segment of $C$ and if $\beta$ denotes the length of the positively oriented segment of $C$ in $\partial D$, then $A(C)=\frac{1}{4} \sin \alpha+\frac{1}{2} \beta$ and $K(C)=\frac{1}{2} \alpha+\beta$. Therefore $K(C)-2 A(C)=$ $\frac{1}{2} \alpha-\frac{1}{2} \sin \alpha \geq 0$.

Summing over all pieces we find that $K\left(\Gamma^{\prime}\right)-2 A\left(\Gamma^{\prime}\right) \geq 0$. This finishes the proof.

\section{Estimates for surface maps}

In this section we use Theorem 3.3 to prove estimates relating total curvature and area of surface maps. We first prove a result for surface maps with stable singularities into $\mathbf{R}^{2}$ and then use this result in combination with standard techniques of integral geometry to obtain similar estimates for maps into $\mathbf{R}^{n}$, $n \geq 2$. 


\subsection{The estimate for maps $f: S \rightarrow \mathbf{R}^{2}$}

We first express the area of a generic smooth map of a surface to a plane through the area content of its fold curve. We say that a map $f: S \rightarrow \mathrm{R}^{2}$ with stable singularities is generic if all multiple points of its fold curve $\Gamma$ are transverse double points (in particular no cusp of $\Gamma$ is a multiple point). It is easy to see that any map with stable singularities may be arbitrarily well approximated by a generic map.

Lemma 4.1. Let $S$ be a closed surface, let $f: S \rightarrow \mathrm{R}^{2}$ be a map with stable singularities, and let $(x, y)$ be orthonormal coordinates on $\mathbf{R}^{2}$. Let $\Sigma$ be the fold curve of $f$ cooriented by the normal field which points towards lower multiplicity, see Figure 8. Then

$$
\operatorname{Area}(f)=\int_{\Sigma}(x d y-y d x) .
$$

Proof. An approximation argument shows that it is enough to show the lemma for generic maps. Thus assume $f$ is generic and let $2 m$ be the highest multiplicity of the map $f$. When a point in $\mathbf{R}^{2}$ crosses $\Sigma$, the multiplicity of $f$ changes by 2 . We split the fold curve $\Sigma$ at its double points as indicated in Figure 8 . The result is finitely many pairwise not intersecting closed curves $C_{i}$ (without self intersections but possibly with cusps-singularities) cooriented so that the coorienting normal points towards regions of lower multiplicity of $f$.
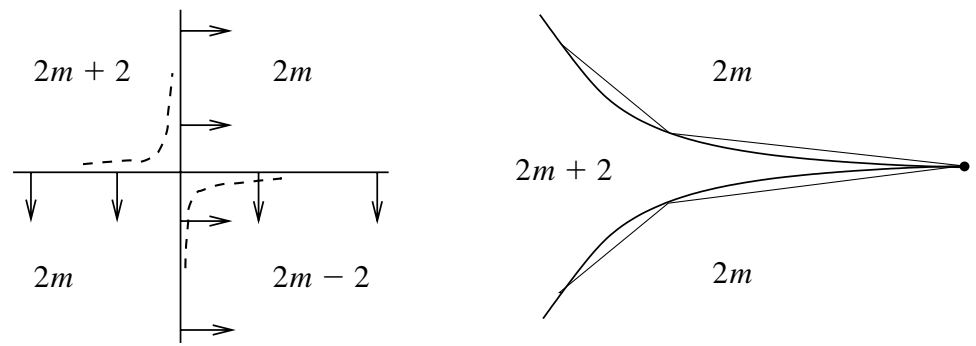

FigURE 8

Let $C(2 k, 2 k-2)$ denote the union of those $C_{i}$ which have a neighborhood $U \subset \mathrm{R}^{2}$ such that for any $p \in U-C_{i}$ the multiplicity of $f$ at $p$ equals $2 k-2$ or $2 k$. Let $A(2 k, 2 k-2)$ denote the area of the subset $W \subset \mathbf{R}^{2}$ which has $C(2 k, 2 k-2)$ as its cooriented boundary. (Note that the multiplicity of $f$ at each point $p \in W$ is $\geq 2 k$.) Then

$$
\operatorname{Area}(f)=\sum_{k=1}^{m} 2 k(A(2 k, 2 k-2)-A(2 k+2,2 k))=2 \sum_{k=1}^{m} A(2 k, 2 k-2) \text {. }
$$


By Stokes theorem,

$$
A(2 k, 2 k-2)=\frac{1}{2} \int_{C(2 k, 2 k-2)}(x d y-y d x) .
$$

Since $\Sigma$ is the disjoint union of $C(2 k, 2 k-2), k=1,2, \ldots, m$ the lemma follows.

We are now ready to state and prove the main theorem of the paper.

Theorem 4.2. For any $C^{3}$ map with stable singularities $f: S \rightarrow \mathbf{R}^{2}$ of a closed surface $S$ with image contained in the disk of radius $\epsilon$

$$
K(f) \geq \frac{\operatorname{Area}(f)}{\epsilon^{2}} .
$$

Proof. By Lemma 4.1 and the argument in Section 2.2 we must show that

$$
K(\Sigma) \geq \frac{1}{\epsilon^{2}} \int_{\Sigma}(x d y-y d x),
$$

where $\Sigma$ is the fold curve of $f$. To this end, note that $\Sigma$ can be approximated by a fold-approximation $\Gamma$ (for the definition, see Section 3 ) in such a way that $A(\Gamma)$ approximates $A(\Sigma)$ arbitrarily well, and so that $K(\Gamma)$ is arbitrarily close to $K(\Sigma)$. (For example, we can use a PL fold-approximation $\Gamma$ with a wedge in each of the cusps of the fold curve $\Gamma$. Note that the curvature of some piece of $\Sigma$ near the cusp is exactly the inner angle at the wedge, see Figure 8.) The theorem then follows from Theorem 3.3.

\subsection{The estimate for $f: S \rightarrow \mathrm{R}^{n}, n>2$}

We prove the counterpart of Theorem 4.2 for $C^{2}$-surface maps into $\mathrm{R}^{n}, n \geq 3$. In order to weaken the differentiability assumption (the maps are assumed $C^{3}$ in Theorem 4.2) we start out with an approximation lemma.

If $f: S \rightarrow \mathrm{R}^{n}$ is a $C^{2}$-map of a surface $S$ with stable singularities then for each point $p \in S$ there exist local coordinates $(u, v)$ around $p \in S$ and $(x, y, z)$ around $f(p)$ in $\mathrm{R}^{3}$ such that $f$ has one of the following local forms.

(a) A regular point $(u, v) \mapsto(u, v, 0)$.

(b) A Whitney umbrella point $(u, v) \mapsto\left(u, u v, v^{2}\right)$.

If $f: S \rightarrow \mathrm{R}^{n}, n \geq 4$, is a $C^{2}$-map of a surface $S$ a with stable singularities then $f$ is an immersion.

Lemma 4.3. Let $S$ be a closed surface and let $f: S \rightarrow \mathbf{R}^{n}, n \geq 3$, be a $C^{2}$ map with stable singularities. Then $f$ can be arbitrarily well approximated in 
the $C^{2}$-topology by a $C^{\infty}$-map with stable singularities. Moreover, if $f_{k}: S \rightarrow$ $\mathbf{R}^{n}, k=1,2, \ldots$ is any sequence of smooth maps which converges to $f$ in the $C^{2}$-norm then

$$
\begin{aligned}
& \operatorname{Area}\left(f_{k}\right) \rightarrow \operatorname{Area}(f) \text { and } \\
& \hat{K}\left(f_{k}\right) \rightarrow \hat{K}(f) \text { as } j \rightarrow \infty .
\end{aligned}
$$

Proof. The approximation statement is well-known. The convergence in (8) is immediate (in fact $C^{1}$-convergence of the maps is sufficient). The same argument, but considering instead of the map $f$ itself its Gauss-map, see Section 2.1, gives the convergence in (9) in case $f$ is an immersion.

The only remaining case is thus when $f: S \rightarrow \mathrm{R}^{3}$ is a map with a finite number of Whitney umbrella points $\left\{s_{1}, s_{2}, \ldots, s_{m}\right\}$, say. Fix a Riemannian metric on $S$. For $\epsilon>0$, let $S_{\epsilon}$ denote the open surface $S-\left(\cup_{j=1}^{m} B\left(s_{j}, \epsilon\right)\right)$ where $B\left(s_{j}, \epsilon\right)$ is the closed ball of radius $\epsilon$ around $s_{j}$.

For $v \in \mathrm{S}^{2}$, let $c_{\epsilon}(v)$ be the number of critical points of the height function $h_{v}: S_{\epsilon} \rightarrow \mathrm{R}, h_{v}(s)=\langle f(s), v\rangle$, see Section 2.1. Clearly, $c_{\epsilon}(v)$ is nondecreasing in $\epsilon$ for any $v$. Moreover, $\lim _{\epsilon \rightarrow 0} c_{\epsilon}(v)=c(v)$ for almost every $v$, since the point $s_{j}$ is a critical point of $h_{v}$ only for $v$ in the great circle $G \subset \mathrm{S}^{2}$ of vectors orthogonal to the line $\operatorname{Im}\left(d f\left(s_{j}\right)\right)$, which is a set of measure 0 . We conclude

$$
\lim _{\epsilon \rightarrow 0} \int_{v \in \mathrm{S}^{2}} c_{\epsilon}(v) d v=\int_{v \in \mathrm{S}^{2}} c(v) d v=K(f) .
$$

For $k$ sufficiently large the number of Whitney umbrella points of $f_{k}$ equals $m$. Also, the Whitney umbrella points of $f_{k}$ converges to those of $f$. Note that both area and total curvature are unaffected by right composition with diffeomorphisms. Thus, after composing $f_{k}$ on the right with $C^{\infty}$-diffeomorphisms $\phi_{k}: S \rightarrow S\left(\phi_{k} \rightarrow\right.$ id as $\left.k \rightarrow \infty\right)$ we may assume that the Whitney umbrella points of all the $f_{k}$ are exactly $\left\{s_{1}, \ldots, s_{m}\right\}$. Letting $c_{\epsilon}^{k}$ be defined as $c_{\epsilon}$ with $f_{k}$ replacing $f$ we conclude from the above results for immersions that

$$
\lim _{k \rightarrow \infty} \int_{v \in \mathrm{S}^{2}} c_{\epsilon}^{k}(v) d v=\int_{v \in \mathrm{S}^{2}} c_{\epsilon}(v) d v .
$$

Equations (10) and (11) together imply (9).

THeorem 4.4. Let $S$ be a closed surface and let $f: S \rightarrow \mathrm{R}^{n}, n \geq 3$, be a $C^{2}$-map with stable singularities and with image contained in the ball of radius $\epsilon$. Then

$$
2 \pi \hat{K}(f) \geq \frac{\operatorname{Area}(f)}{(n-1) \epsilon^{2}} .
$$


Proof. Lemma 4.3 shows that it is sufficient to prove the theorem for smooth maps $f$ with stable singularities. We proceed by induction on the dimension $n$. For smooth $f$ the theorem holds for $n=2$ by Theorem 3.3. Assume that the theorem holds for all maps $g: S \rightarrow \mathrm{R}^{n-1}$ with stable singularities. Let $v \in \mathbf{S}^{n-1} \subset \mathbf{R}^{n}$, let $\pi_{v}: \mathbf{R}^{n} \rightarrow \mathrm{R}^{n-1}$ denote orthogonal projection to the hyperplane orthogonal to $v$, and let $d \sigma$ denote the rotationally invariant measure on $\mathrm{S}^{n-1}$ of total mass 1 . A standard transversality argument shows that $\pi_{v} \circ f: S \rightarrow \mathrm{R}^{n-1}$ has stable singularities for almost every $v \in \mathbf{S}^{n-1}$. Moreover, (as is easily verified)

$$
\int_{v \in S^{n-1}} \operatorname{Area}\left(\pi_{v} \circ f\right) d \sigma=\frac{n-1}{n-2} \operatorname{Area}(f)
$$

and

$$
\hat{K}(f)=\int_{v \in \mathrm{S}^{n-1}} \hat{K}\left(\pi_{v} \circ f\right) d \sigma .
$$

The theorem follows.

\subsection{Best constants}

Let us conclude with remarking that for $n=3$, equality in (12) holds for the standard 2-sphere of radius $\epsilon$. Orthogonal projection of this sphere to a plane gives equality in (7). Hence, in the first two dimensions the estimate is sharp. For $n>3$, we do not know of any example which gives equality in (12). We therefore ask: what is the best constant in the estimate of Theorem 4.4 for $n \geq 4$ ?

\section{REFERENCES}

1. Arnold, V. I., Gusein-Zade, S. M., Varchenko, A. N., Singularities of Differentiable Maps, Vol. 1, Monogr. Math. 82 (1985).

2. Aminov, Ju. A., Estimates of the diameter and volume of a submanifold of a Euclidean space, Ukrain. Geom. Sb. 18 (1975), 3-15.

3. Burago, Yu. D., Zalgaller, V. A., Geometric Inequalities, Translated from the Russian by A. B. Sosinski1. Grundlehren Math. Wiss. [Fundamental Principles of Mathematical Sciences], 285. Springer Series in Soviet Mathematics (1988).

4. Cecil, T. E., Ryan, P. J., Tight and taut immersions of manifolds, Pitman Res. Notes Math. Ser. 107 (1985).

5. Milnor, J. W., On the total curvature of knots, Ann. of Math. 52 (1950), 248-257.

6. Fáry, I., Sur certaines inégalites géométriques, Acta Sci. Math. Szeged 12, Leopoldo Fejer et Frederico Riesz LXX annos natis dedicatus, Pars A, (1950), 117-124.

7. Santaló, L. A., On some geometric inequalities in the style of Fáry, Amer. J. Math. 91 (1969), 25-31.

8. MR, Review 39\#3395. 
9. Zbl., Review 0176.19606.

UPPSALA UNIVERSITY

BOX 480

SE-751 06 UPPSALA

SWEDEN

E-mail: tobias@math.uu.se
MID SWEDEN UNIVERSITY

SE-851 70 SUNDSVALL

SWEDEN

E-mail: frank.kutzschebauch@mh.se 